P160 (continued)

engage different intelligences; and tailoring activity duration to be developmentally appropriate. Pragmatic considerations included: essential elements, such as developing culturally relevant educational materials; and logistics, such as working within the financial, time, and space constraints of community-based afterschool programs.

Conclusions and Implications: The insight gained from this study lays the foundation for understanding the necessary components for well-implemented and effective interventions for youth-focused NE programs in community-based afterschool settings.

Funding: None

\section{P161 In Defense of Food Curriculum: Investigating Students' Understanding and Application of Food Rules in their Lives}

Pamela Koch, EdD, RD, pak14@tc.columbia.edu, Teachers College, Columbia University, 525 West 120th Street Box 137, New York, NY 10027; H. Bhana, MHSc;

L. Sienkiewicz, BS; H. L. Gray, PhD, RD; I. Contento, PhD

Objective: The In Defense of Food (IDOF) Curriculum provides students with tools to eat a nutritious diet, despite our complex, confusing food environment. This study examined if learning practical, actionable messages through Michael Pollan's Food Rules increased students awareness and understanding.

Design, Setting, and Participants: Post-only design with 22 middle school students in sixth and seventh grade, primarily African American and Hispanic, living in a lowincome, urban neighborhood.

Outcome Measures and Analysis: After receiving the IDOF Curriculum, student were asked to state the meaning of the 11 Food Rules they learned in their own words, and to provide examples of how to apply it in their lives. The researcher who conducted the interviews wrote down students' responses verbatim. Each response was scored on a three-point scale, $1=$ no understanding to $3=$ full understanding. Descriptive statistics calculated mean scores for each Food Rule. Qualitative analysis determined themes by Food Rule and by the three big messages of the curriculum (Eat food. Not too Much. Mostly Plants.)

Results: Students had good understanding of the Food Rules with $54 \%$ of responses receiving a score of 3 . For these responses students were able to accurately describe and apply the Food Rule. The mean scores for each Food Rule ranged from 1.73 to 2.68 . The qualitative analysis overall indicated that the Food Rules supported the students in understanding and applying the three big messages.

Conclusions and Implications: These findings indicate that learning the Food Rules in IDOF appeared to be an effective way to guide students toward eating better. Further studies with a larger randomized controlled designs are warranted.

Funding: National Science Foundation
P162 'Let's Work Together Towards Children's Nutrition': Building Bridge Between Child Care Providers and Parents for Promoting Child Health

Dipti Dev,PhD, ddev2@unl.edu, University of Nebraska, Lincoln, 1400 R Street, Lincoln, NE 68588; C. ByrdWilliams, PhD, University of Texas; S. Ramsay, PhD, University of Idaho; $B$. McBride, PhD, University of Illinois at Urbana-Champaign; $A$. May, PhD, Centers for Disease Control; C. Arcan, PhD, Univerisity of Minnesota; A. M. Adachi- Mejia, PhD, Dartmouth Institute for Health Policy \& Clinical Practice

Objective: To investigate childcare providers' perspectives for communicating with parents to promote children's nutrition and health.

Design, Setting, and Participants: In-person, semistructured interviews. Licensed center-based child care. Eighteen full-time childcare providers caring for children 2-5 years old, $67 \%$ caring for children who pay with federal subsidy.

Outcome Measures and Analysis: Participants were selected using maximum variation purposive sampling. Interviews were conducted until saturation was achieved. NVivo, thematic analysis used to code and identify themes and meta-themes regarding providers' perspectives for parent communication.

Results: Two meta-themes emerged from providers' responses: barriers to communication, and strategies to communication. Barriers to communication include: parents are too busy to talk, parents prioritize food concerns over nutrition, parents allow unhealthy foods, providers are unsure about communicating, and providers weren't sure parents were receptive to nutrition education materials. Five strategies were identified for communication and building bridges between providers and parents: ecognize the benefits of engaging and educating parents about nutrition to promote health; everage policy to communicate positively and avoid conflict with parents; implement center-level practices to reinforce policy; build partnerships with parents through education; foster a respectful relationship between providers and parents.

Conclusions and Implications: Policy and center strategies were identified for fostering a respectful relationship and building bridges between providers and parents to promote child health and nutrition.

Funding: Administration for Children and Families Office of Planning and Evaluation Research, Illinois Transdisciplinary Obesity Prevention Program (I-TOPP)

\section{P163 Evaluation of the Effectiveness of the Drexel University Eat.Right.Now. High School Curriculum in Pennsylvania SNAP-Ed}

Judy Ensslin, MS, RD, LDN, jae58@drexel.edu, Drexel University, 3141 Chestnut Street, Philadelphia, PA 19104; A. Marsteller, MBA, MS, RDN, LDN; S. L. Volpe, PhD, RD, LDN, FACSM

Objective: To demonstrate a positive impact on eating behaviors in Philadelphia high school students after

Continued on page $S 72$ 


\section{P163 (continued)}

receiving nutrition education utilizing the Drexel University High School Curriculum.

Target Audience: High school students from SNAP-Ed eligible schools in the School District of Philadelphia assigned to Drexel University's Eat.Right.Now. Nutrition Education Program.

Theory, Prior Research, Rationale: SNAP-Ed guidance requires the use of evidence-based nutrition education interventions. Minimal evidence-based curricula for high school students exist. This evaluation will help to establish an evidence-based, high school curriculum for SNAP-Ed.

Description: High schools were randomly selected from SNAP-Ed eligible high schools receiving Drexel University's Eat.Right.Now. Program. The intervention utilized the modified Youth Risk Behavior Survey (YRBS). Students received a baseline survey followed by a five-lesson series using the Drexel University High School Curriculum, and a post-intervention survey. A total of 1100 baseline and 972 post-intervention surveys were completed.

Evaluation: Baseline and post-intervention surveys were compared to the 2011 YRBS National results for high school students. At baseline, less students reported eating fruit daily ( $\mathrm{p}=0.0078)$, more reported not eating vegetables $(\mathrm{p}=0.0083)$, and more students reported soda consumption $(\mathrm{p}=0.0138)$ than National YRBS respondents. Postintervention results demonstrated an elimination of the significance for fruit and vegetable intake, a greater intake of vegetables $(\mathrm{p}=0.0152 ; \mathrm{p}<0.0001)$, and significantly more students not drinking soda $(\mathrm{p}=0.0079)$ compared to National YRBS respondents.

Conclusions and Implications: Our intervention positively affected eating behaviors of high school students receiving our curriculum. The Drexel University Eat.Right.Now. High School Curriculum is an effective tool to teach students healthy eating behaviors.

Funding: Supplemental Nutrition Assistance Program Education

\section{P164 Online Game to Support Nutrition Education}

Stephanie Folkens, BS, AAS, sfolkens@commonthreads.org, Common Threads, 3811 Bee Caves Road, Suite 108, Austin, TX 78746; J. Necessary, BS; L. Ferrari, BS, Octobot; J. Stetson Pastore, MS, UXRD; N. K. Edens, PhD, Common Threads

Objective: CommonBytes (CB) is an online game from Common Threads (CT) aiming to improve nutrition and cooking literacy through increasing program fidelity and support for nutrition education and digitally connecting participants.

Target Audience: 3 rd-8th grade students and classroom teachers.

Theory, Prior Research, Rationale: CT trains and funds over 1,000 classroom teachers annually to implement nutrition education. A digital resource is frequently requested and of $141 \mathrm{CT}$ teachers surveyed, 72\% indicated they would use an online game to support nutrition educa- tion. $67 \%$ of the teachers indicated students would use the game in group or individual work. CT worked with UXRD Inc. (Miami) and Octobot (San Francisco) to build CB. UXRD followed a user-centered product development methodology (ISO 9241-210:2010 Human-Centered Design for Interactive Systems) and the Design for Kids framework to build wireframes. Octobot built CB from the wireframes. $\mathrm{CB}$ is being piloted at 9 Miami schools and in 2016 will roll out in 6 other districts.

Description: $\mathrm{CB}$ includes 12 games with three levels. The games bring gardening, grocery shopping, and cooking to nutrition education with 45 recipes. As they play, participants earn points for themselves, their class, school, and district. Parents and teachers can also track student progress.

Evaluation: With IRB and RRC approval, the website and Google Analytics record data for improving CB. Focus groups with students and teachers will identify areas for improvement as CB is developed further.

Conclusions and Implications: $C B$ provides an online platform to teach and support nutrition education. Public access in 2016 will generate data to help understand digital learning and behaviors in this field.

Funding: None

\section{P165 On the Move to Better Health Junior Promotes Behavior Change}

Julie Garden-Robinson, PhD, RD, LDN, julie.

garden-robinson@ndsu.edu, North Dakota State

University, 1340 Administration Avenue, Fargo, ND 58102

Objective: The curriculum is designed to improve knowledge of food and nutrition concepts, to increase intake of fruits and vegetables, and to increase physical activity.

Target Audience: Children (average age of 8 years old) and their parents have participated.

Theory, Prior Research, Rationale: Using principles of social cognitive theory, the curriculum employs experiential learning, role modeling and environmental cues to promote behavior change.

Description: Five weekly lessons were implemented in second grade classrooms. Students learned to identify foods from different food groups and "tracked" their daily intake of one food group per week on a simple coloring chart. Children received small weekly incentives, such as colorful pencils and erasers. Parents received weekly newsletters and family goal-setting sheets.

Evaluation: Nationally-approved pre- and post- picturebased surveys assessed knowledge gain and achievement of objectives. Participating boys and girls $(n=1,260)$ improved their ability to identify healthful snacks $(\mathrm{p}<.05)$. About $87 \%$ of participating children reported trying a new food, $89 \%$ ate more fruit, $77 \%$ ate more vegetables, $78 \%$ tried different fruits of different colors, $68 \%$ tried vegetables of different colors, $92 \%$ got more exercise, and $98 \%$ had fun. According to parent survey results $(n=328), 83 \%$ of parents read the weekly newsletter. Parents reported that their children asked questions about food (54\%), offered help with food preparation (48\%), requested healthful snacks (53\%), and tried a new food (45\%).

Continued on page S73 